04

\title{
Резонансные свойства диэлектрического барьерного разряда при пониженном давлении
}

\author{
() В.М. Бочарников, ${ }^{1}$ В.В. Володин, ${ }^{1}$ В.В. Голуб,, И.В. Трифранов, ${ }^{1,2}$ Д.Г. Серебряков ${ }^{1,2}$ \\ ${ }^{1}$ Объединенный институт высоких температур РАН, \\ 125412 Москва, Россия \\ ${ }^{2}$ Московский фризико-технический институт (НИУ), \\ 141700 Долгопрудный, Московская обл., Россия \\ e-mail: vova.m.bocha@gmail.com
}

Поступило в Редакцию 25 февраля 2020 г.

В окончательной редакции 2 апреля 2020 г.

Принято к публикации 17 апреля 2020 г.

Исследованы электрические характеристики диэлектрического барьерного разряда (ДБР) симметричного актуатора при пониженных давлениях. Проведен анализ системы „источник питания-актуатор-ДБР“. Построены зависимости частоты питающего напряжения, при которых наблюдается резонанс напряжения на внешних электродах актуатора заданной геометрии, от давления воздуха. Определены мощность ДБР, емкость и добротность системы в зависимости от давления в барокамере. Обнаружено, что при уменьшении давления доля энергии ДБР, расходуемая на создание синтетической струи, существенно увеличивается.

Ключевые слова: диэлектрический барьерный разряд, симметричный актуатор, синтетическая струя.

DOI: $10.21883 /$ JTF.2020.12.50122.63-20

В последние годы диэлектрический барьерный разряд (ДБР) активно исследуется в качестве способа управления потоком вокруг плоскостей летательного аппарата $[1,2]$. Для этого используют симметричные и асимметричные актуаторы. Асимметричный актуатор создает пристеночную струю, направленную вдоль поверхности актуатора [3]. Если установить два асимметричных актуатора друг по направлению к другу с общим инкапсулированным электродом, то при взаимодействии пристеночных струй образуется течение, направленное перпендикулярно поверхности актуатора. Поскольку рабочее тело для такого течения поступает с обтекаемой поверхности, полученную струю можно классифицировать как „синтетическую“ [4,5]. Такого рода струя может быть использована не только для предотвращения и затягивания отрыва, но и как закрылок. При этом возможно управлять подъемной силой и создавать моменты по крену и тангажу. Для повышения эффективности этого способа необходимо, чтобы управляющая струя обладала наибольшим импульсом при минимальных затратах энергии. Синтетическая струя возникает в процессе ДБР, и ее тяга сушественно зависит от свойств разряда. ДБР условно можно разделить на два типа: диффузионный и стримерный [6,7]. Для создания синтетических струй большей тяги диффузионный режим является предпочтительным [8,9]. На режим разряда влияют как электрические параметры источника питания и актуатора, так и геометрия актуатора и давление окружающего воздуха $[10,11]$. В настоящей работе на примере простейшего линейного симметричного актуатора были определены резонансные характеристики разряда и системы в целом в зависимости от геометрии актуатора и давления воздуха. На основании экспериментальных данных предложено теоретическое объяснение повышения эффективности создания синтетической струи с помощью ДБР при снижении давления воздуха.

Схемы экспериментальной установки и симметричного актуатора представлены на рис. 1. Экспериментальная установка состояла из ДБР актуатора, барокамеры, источника высокого напряжения и средств измерения. Актуатор представлял собой диэлектрическую пластину с электродами. На пластину приклеивался инкапсулированный электрод 1 , выполненный из полосы алюминиевой фольги толщиной $50 \mu \mathrm{m}$ и шириной от 2 до $6.5 \mathrm{~cm}$. На инкапсулированный электрод наклеивался диэлектрик 2 из лавсана толщиной $H=350 \mu \mathrm{m}$. На диэлектрик - внешние электроды 3, также выполненные из полосы алюминиевой фольги толщиной $h=50 \mu \mathrm{m}$ и шириной $0.5 \mathrm{~cm}$. Длина электродов $L$ варьировалась от 4 до $20 \mathrm{~cm}$. Расстояние между внешними электродами $d$ всегда было равно ширине инкапсулированного электрода. Актуатор помещался в барокамеру, оборудованную вакуумным насосом и вакуумметром. Через гермоввод в барокамере к актуатору подводилось высокое синусоидальное напряжение с амплитудой $6.5-7 \mathrm{kV}$ и частотой $1.5-5 \mathrm{kHz}$. Мощность, вкладываемая в разряд, и его эффективная емкость измерялись при помощи вольткулоновской характеристики. Методика измерения мощности и емкости ДБР детально изложена в [12]. После установки актуатора барокамера закрывалась и откачивалась до нужного давления. После включения высокого напряжения частота подбиралась таким образом, чтобы амплитуда напряжения на внешних электродах актуатора была максимальной при неизменной выходной мощ- 


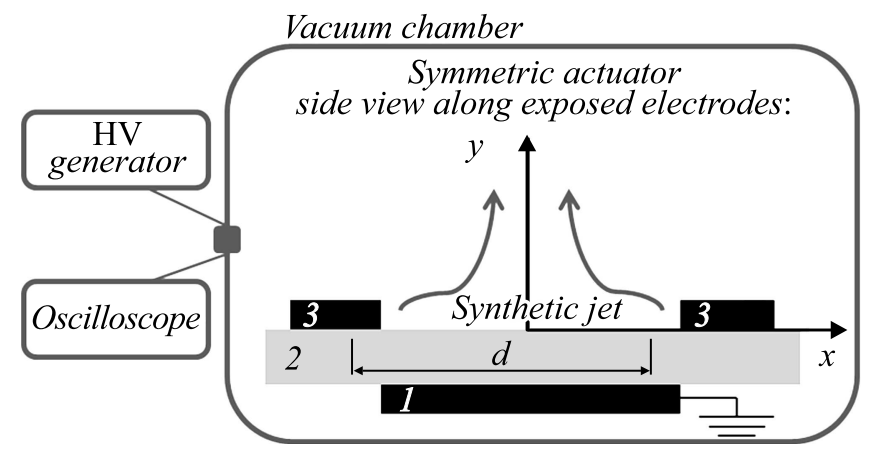

Рис. 1. Схема экспериментальной установки и симметричного ДБР актуатора: 1 - инкапсулированный электрод, 2 - диэлектрик, 3 - внешние электроды.

ности источника. Источником высокого переменного напряжения служил генератор звуковой ГЗ-33 в сочетании с транзисторным усилителем, питаемым источником постоянного напряжения AKTAKOM APS 1503 и масляной катушкой зажигания ТУ 37.466.072-96. Напряжение высоковольтного сигнала контролировалось осциллографом RIGOL DS1054Z с делительным щупом 1-1000. Сила тока через актуатор контролировалась с помощью конденсатора в разрыве цепи актуатор-генератор по методике, описанной в работе [12]. Постоянство выходной мощности обеспечивалось подбором напряжения высокочастотного сигнала и напряжения питания усилителя. Мощность сигнала от звукового генератора значительно меньше мощности источника постоянного напряжения, поэтому за выходную мощность источника принималась мощность питания усилителя. Частоты, на которых проводились эксперименты, близки к оптимальным для масляной катушки зажигания ТУ 37.466.072-96. Относительная погрешность измерения выходной мощности не превышала 3\%. Абсолютные погрешности при измерении давления в барокамере и амплитуды напряжения на внешних электродах составляли 1.5 Torr и $5 \mathrm{~V}$ соответственно, поэтому в дальнейшем планки этих погрешности не будут обозначаться.

КПД определялся при резонансе напряжения на внешних электродах, так как при большем напряжении достигается большая тяга синтетической струи [13], что важно для практического применения актуаторов. КПД диэлектрического барьерного разряда вычислялся следующим образом:

$$
\eta=\frac{P_{\mathrm{DBD}}}{P_{\text {in }}}
$$

где $P_{\mathrm{DBD}}-$ мощность, вкладываемая в ДБР, $P_{i n}-$ выходная мощность источника. Мощность ДБР определяется по напряжению на внешних электродах и току разряда. Средняя мощность потерь энергии в диэлектрике составляла не более $5 \mathrm{~mW}$ из-за высокого удельного сопротивления лавсана, что составляло малую долю от мощности ДБР, поэтому не будет учитываться в дальнейшем.
При снижении давления в барокамере обнаружено уменьшение резонансной частоты (рис. 2). Резонансная частота определяется электрической емкостью диэлектрического барьерного разряда и индуктивностью источника питания. Индуктивностью разряда можно пренебречь по сравнению с индуктивностью катушки, так как магнитное поле, создаваемое током разряда, существенно отлично от нуля лишь на краях актуатора и не создает магнитного потока в проводнике. Так же стоит упомянуть, что наибольший ток может быть лишь в контрагированных филаментах [14], которые при снижении давления не формируются, т.е. разряд становится более диффузионным. Фотографии разряда с экспозицией $1 \mathrm{~s}$ представлены на рис. 3, фотографии с меньшей экспозицией, позволяющей увидеть наличие контрагированных филамент при высоком давлении и их отсутствие при низком, можно увидеть, например, в работах [15-17]. Так как индуктивность источника питания неизменна, следовательно, изменение резонансной частоты показывает существенное изменение свойств разряда.

На рис. 4 представлены резонансные кривые для симметричного актуатора с внешними электродами длиной $15 \mathrm{~cm}$ и расстоянием между ними $d=6.5 \mathrm{~cm}$ при различных давлениях в барокамере. Пик резонанса довольно сильно размыт из-за того, что изменение частоты связано с изменением как амплитуды напряжения, так и с коэффициентом диссипации энергии. ДБР - многопараметрическая система, и при его исследовании можно поддерживать на одном уровне ограниченное количество величин, как то входная мощность, частота, амплитуда питающего напряжения, давление в барокамере. Изменение одной из характерных величин влечет за собой комплексную перестройку всей системы [18]. Из рис. 4 видно, что при снижении давления уменьшается резонансная амплитуда напряжения на внешних электродах. Таким образом, идет подстройка запасаемой в системе

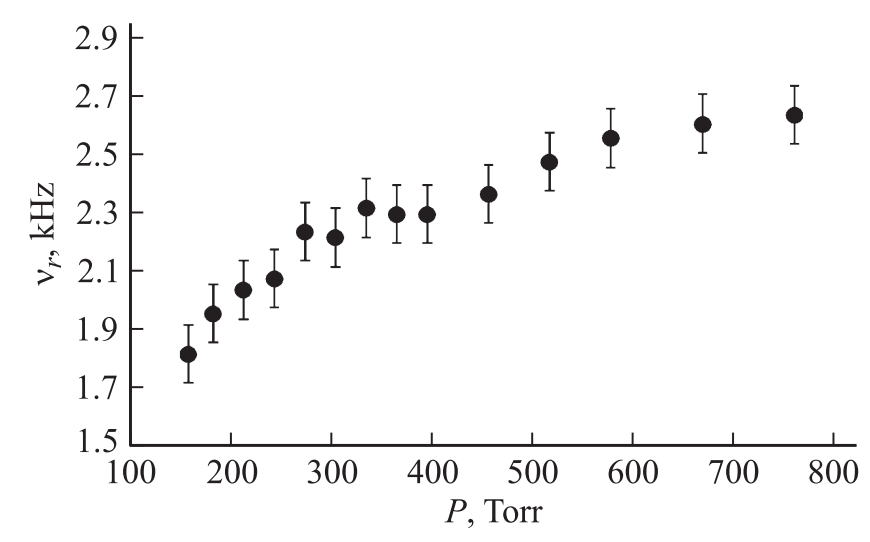

Рис. 2. Зависимость резонансной частоты от давления. Симметричный актуатор, длина внешних электродов $15 \mathrm{~cm}$, расстояние между ними $6.5 \mathrm{~cm}$. 

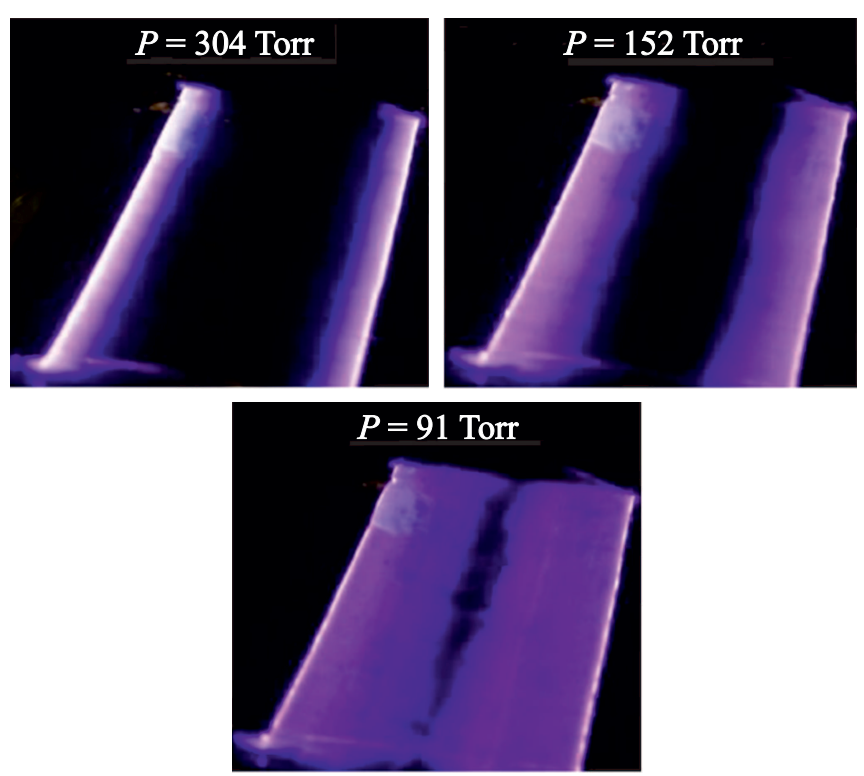

Pис. 3. Фотографии диэлектрического барьерного разряда при различном давлении в барокамере с экспозицией $1 \mathrm{~s}$.

энергии под изменяющуюся емкость:

$$
C=\frac{q_{s}}{U}
$$

где $C$ - емкость системы, $q_{s}$ - заряд на поверхности диэлектрика, $U$ - напряжение на внешних электродах. Эта формула связывает емкость системы, напряжение на внешних электродах и накопленный на диэлектрике поверхностный заряд. Максимальная величина накопленного заряда зависит от площади плазменного листа [19] и соответственно от давления в барокамере, как будет показано ниже.

На рис. 5 представлены измеренные зависимости КПД разряда от давления при различных значениях длины внешних электродов и фиксированной выходной мощности источника, равной $30 \mathrm{~W}$. КПД разряда повышается при снижении давления в барокамере и при увеличении длины внешних электродов. Для объяснения полученной зависимости следует определить соотношение мощности, расходуемой на создание синтетической струи к мощности, потребляемой на вспомогательные процессы. Энергия диэлектрического барьерного разряда расходуется на выделение тепла $P_{h}$, ионизацию воздуха $P_{i}$ и ускорение газа в направлении, перпендикулярном плоскости актуатора, $P_{m}$. Таким образом, мощность ДБР можно представить следующим образом:

$P_{\mathrm{DBD}}=P_{h}+P_{m}+P_{i}=\int j_{e} E_{x} d V+\frac{2}{\tau} \int e n_{n} E_{y} d y+P_{i}$,

где $j_{e}-$ плотность тока электронов, $T-$ период питающего напряжения, $n_{n}$ - концентрация отрицательных ионов. В первом интеграле во внимание принимаются только электроны, так как их подвижность на два порядка выше, чем подвижность ионов. Интегрирование проводится по объему $V=L \cdot h \cdot l$, где $l$ - длина распространения разряда вдоль поверхности диэлектрика в направлении, перпендикулярном внешним электродам. За пределами указанного объема ток электронов незначителен. Во втором интеграле во внимание принимаются лишь отрицательные ионы, так как объемная сила при отрицательной полярности электродов на порядок превосходит объемную силу при положительной полярности. Величину напряженности электрического поля можно разделить на две составляющие: $E_{x}$ - параллельную поверхности актуатора и перпендикулярную краям внешних электродов и $E_{y}-$ направленную по нормали к поверхности актуатора. Из-за того, что $h \ll l$ можно считать, что электроны, эмитированные с поверхности электрода, движутся только в поле $E_{x}$. $E_{x}$ является суперпозицией электрического поля от внешнего электрода и поля, создаваемого поверхностным зарядом на диэлектрике, и существенно отлично от нуля лишь в катодном слое [20]. $E_{y}$ так же является суперпозицией электрического поля от внешних электродов и поля, создаваемого поверхностным зарядом электронов на

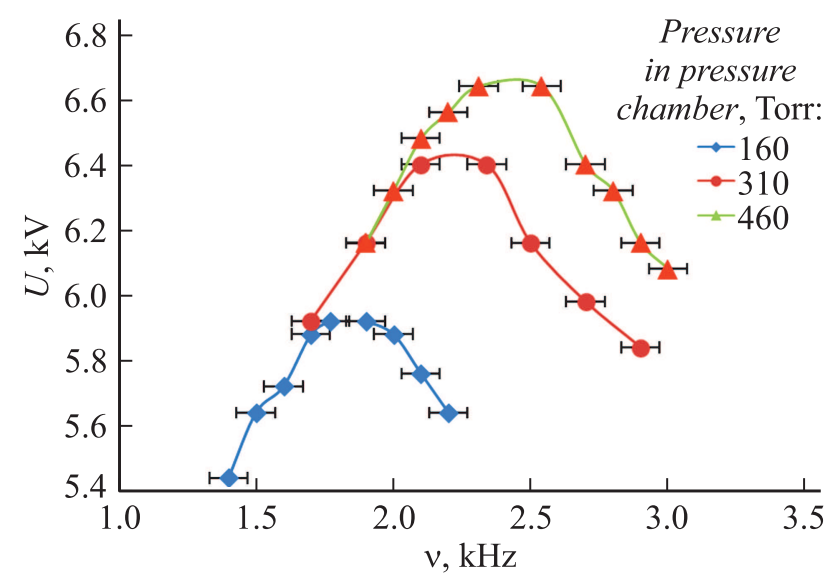

Рис. 4. Резонансные кривые при различных давлениях.

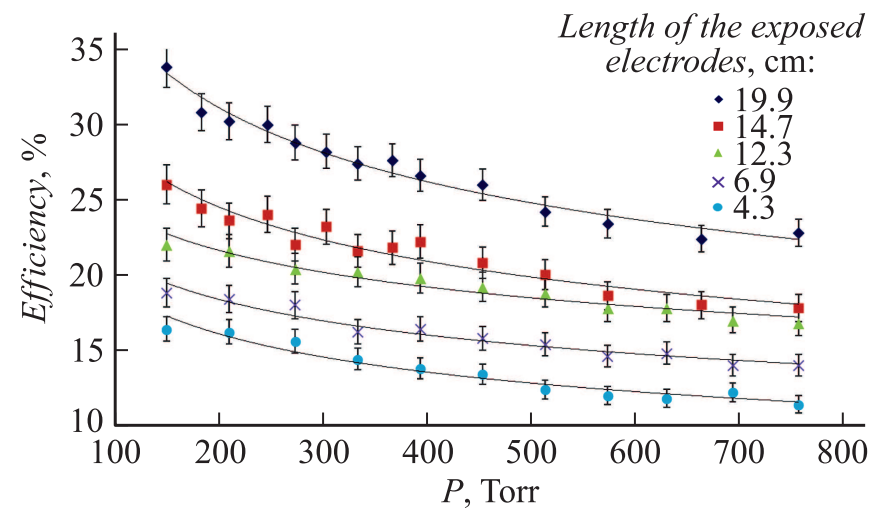

Рис. 5. Зависимость КПД диэлектрического барьерного разряда от давления. Разные зависимости соответствуют разным значениям длины внешних электродов симметричного актуатора. 


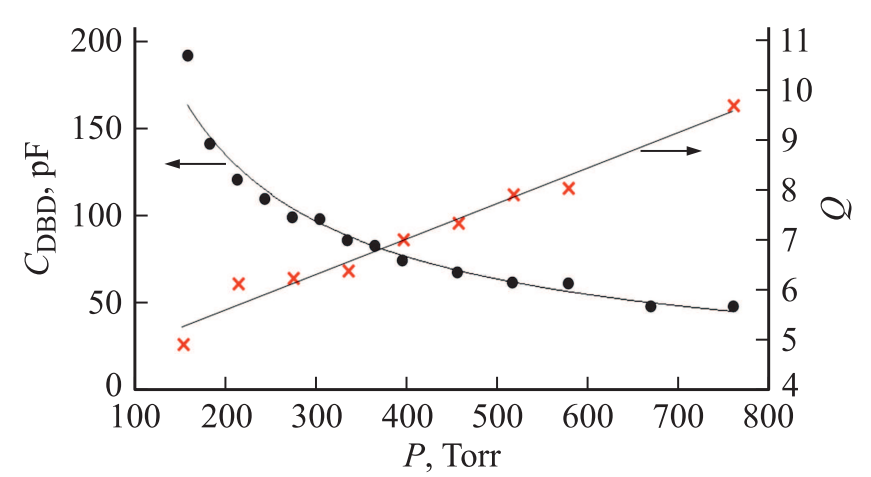

Рис. 6. Зависимость емкости и добротности системы от давления.

диэлектрике. Однако в этом случае они сонаправлены и усиливают друг друга. Так как работа, совершаемая кулоновской силой внешних электродов за период практически равна нулю, основной вклад в импульс струи вносит поле поверхностного заряда. $E_{y}$ является полем, разгоняющим ионы в направлении перпендикулярном поверхности актуатора, и приводит к возникновению синтетической струи в процессе столкновения ионов с нейтралами.

На рис. 6 показаны зависимости емкости и добротности системы от давления для актуатора с внешними электродами длиной $15 \mathrm{~cm}$ и расстоянием между ними $6.5 \mathrm{~cm}$. Погрешности на графиках порядка размера маркера. При уменьшении давления емкость актуатора существенно возрастает. Это вызвано тем фактом, что при уменьшении давления увеличивается длина распространения плазменного листа $l \propto p^{-\frac{i}{z}}[21]$. При нарастании отрицательного потенциала на электродах происходит зарядка поверхности диэлектрика электронами, эмитированными с внешних электродов. Максимальный потенциал, который может иметь поверхность, ограничивается амплитудой напряжения на внешних электродах [22]. Таким образом, при увеличении площади, покрываемой ДБР, увеличивается величина поверхностного заряда, накопленного на диэлектрике, что приводит к повышению эффективной емкости актуатора. Считая разряд однородным вдоль кромки внешних электродов (диффузионный режим), заряд, накопленный на поверхности диэлектрика, можно выразить как $q_{s}=2 \int_{d_{s}}^{l} \sigma d x$, где $\sigma$ - поверхностная плотность заряда, $d_{s}-$ толщина катодного слоя. Заряженную поверхность вместе с инкапсулированным электродом можно представить как плоский конденсатор. Тогда эффективная емкость актуатора будет функцией длины распространения плазменного листа и соответственно давления в барокамере

$$
C=\frac{q_{s}}{U_{1}}=\frac{(\sigma) 2 L i}{U_{1}}=2 \frac{(\sigma)}{U_{1}} L p^{-\frac{i}{2}}
$$

где $U_{1}-$ амплитуда напряжения на внешних электродах, $\langle\sigma\rangle$ - средняя поверхностная плотность заряда на диэлектрике. Из графика на рис. 6 видно, что такое предположение согласуется с экспериментальными данными. Однако экспериментально полученная зависимость емкости от давления аппроксимируется как $C \propto p^{-0.8}$. Расхождение в показателе степени вызвано тем, что совокупность актуатора и ДБР строго не является плоским конденсатором, а накопленный на поверхности диэлектрика заряд распределен неравномерно, и его плотность существенно зависит от расстояния до кромки внешнего электрода. На расстоянии $0.5 d$ происходит экранирование поля от каждой половины симметричного актуатора и в окрестности этой точки $\sigma$ практически обращается в ноль [23].

С увеличением емкости разряда растет максимальная энергия, запасаемая в нем. Расход энергии системой на джоулево тепло и создание синтетической струи является функцией запасенной энергии. Так как ДБР проявляется в виде последовательности микроразрядов [24], средняя разность потенциалов между внешним электродом и поверхностью диэлектрика не изменяется с увеличением длины распространения плазменного листа, а больший поверхностный заряд накапливается за счет увеличения длительности микроразрядов. Таким образом, в соответствии с первым интегралом в (1), при уменьшении давления мощность, затрачиваемая на тепловыделение, практически не изменяется. В то же время, расход энергии на ионизацию возрастает из-за увеличения длины пробега электронов до их оседания на диэлектрике.

$$
P_{i}=\frac{2}{\tau} E_{i} \alpha\langle x\rangle, \quad \alpha=A p \exp \left(-\frac{B p}{E_{x}}\right),
$$

где $p$ - давление воздуха, $A, B-$ константы, $E_{i}-$ эффективная энергия ионизации воздуха, $\langle x\rangle-$ средняя длина пробега электрона до его оседания на поверхности, являющаяся функцией длины распространения разряда. Данный процесс иллюстрируется ростом разности фаз между напряжением и током при уменьшении давления (рис. 7). Результатом увеличения количества электронов в объеме $V$ становится в соответствии с (2) рост количества ионов.

Увеличение поверхностного заряда приводит к увеличению $E_{y}$ и соответственно, величины кулоновской силы, а значит, и работы по разгону ионов электрическим полем. Вследствие этого, при снижении давления мощность диэлектрического барьерного разряда растет за счет увеличения второго и третьего слагаемого в уравнении (1), а добротность системы уменьшается (рис. 6). При уменьшении давления с 760 до 150 Torr добротность уменьшается в два раза. С учетом роста мощности разряда больше энергии рассеивается в системе „актуатор-ДБР“. Данная энергия расходуется преимущественно на увеличение количества ионов и их разгон в поле поверхностного заряда. Таким образом, для практического применения актуаторов в качестве элементов управления потоком вокруг крыльев лета- 
тельного аппарата на высотах до $20 \mathrm{~km}$ можно утверждать, что снижение скоростного напора синтетической струи за счет уменьшения плотности воздуха с высотой компенсируется дополнительной энергией, которую получают ионы между столкновениями с нейтралами.

Кроме КПД можно ввести параметр „приведенный КПД“

$$
\eta^{\prime}=\frac{P_{a c t}}{P_{a c t}+P_{j e t}}
$$

где $P_{a c t}-$ мощность безвозвратных потерь, т.е. совершающих работу, а $P_{j e t}-$ средняя мощность зарядки конденсатора, который представляет собой поверхностный заряд на диэлектрике и инкапсулированный электрод вкупе с изолятором. $P_{\text {jet }}$ меньше, чем $P_{\text {in }}$ из-за дополнительных потерь энергии электронами до их оседания на поверхности диэлектрика, т.е. за счет $P_{h}$ и $P_{i}$ (см. формулу (1)). Так же влияет на процесс и катодная рекомбинация: вторичная электронная эмиссия с поверхности электрода за счет положительных ионов. Таким образом, можно написать уравнение баланса энергии системы (в единицу времени): $P_{j e t}=P_{i n}-P_{h}-P_{i}$. Сравнив эту формулу с (1) можно сделать вывод, что механическая мощность $P_{m}$, т. е. ускорение отрицательных ионов под действием кулоновской силы заряженной поверхности может быть оценена как

$$
P_{m}=P_{j e t}=\frac{2}{\tau} \frac{C U_{1}^{2}}{2}=v_{r} C U_{1}^{2},
$$

где $v_{r}$ - резонансная частота системы, при которой получена вольт-кулоновская характеристика. Для актуатора с электродами длиной $15 \mathrm{~cm}$ при фиксированной питающей мощности $30 \mathrm{~W}$ построена зависимость механической мощности системы от давления (рис. 8). Можно заметить, что при снижении давления в барокамере от $1 \mathrm{~atm}$ до 150 Torr мощность увеличивается в два раза, что согласуется с двукратным уменьшением добротности (рис. 6) и находится в согласии с проведенным ранее анализом. При уменьшении давления уменьшается резонансная частота и, как следствие, скорость роста напряжения на внешних электродах $d U / d t$, которая, в

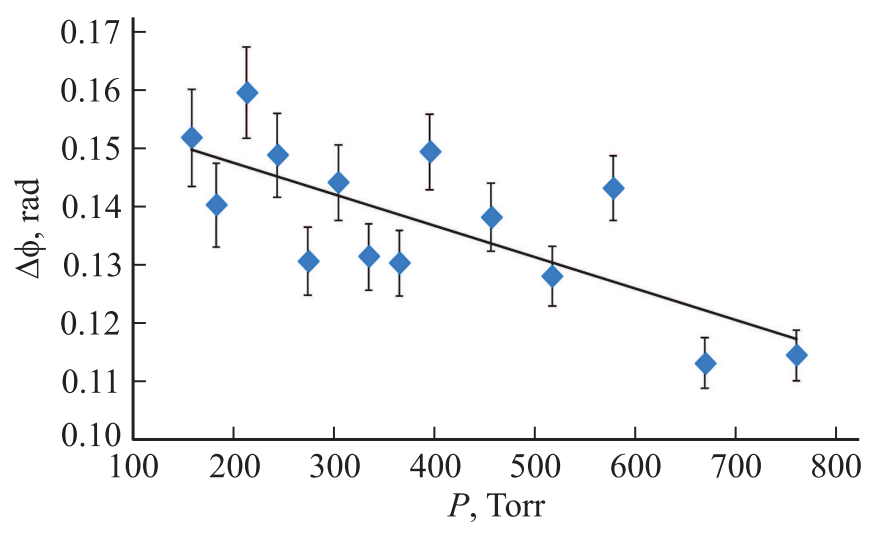

Рис. 7. Зависимость разности фаз между напряжением и током от давления.



Рис. 8. Зависимости механической мощности от давления.

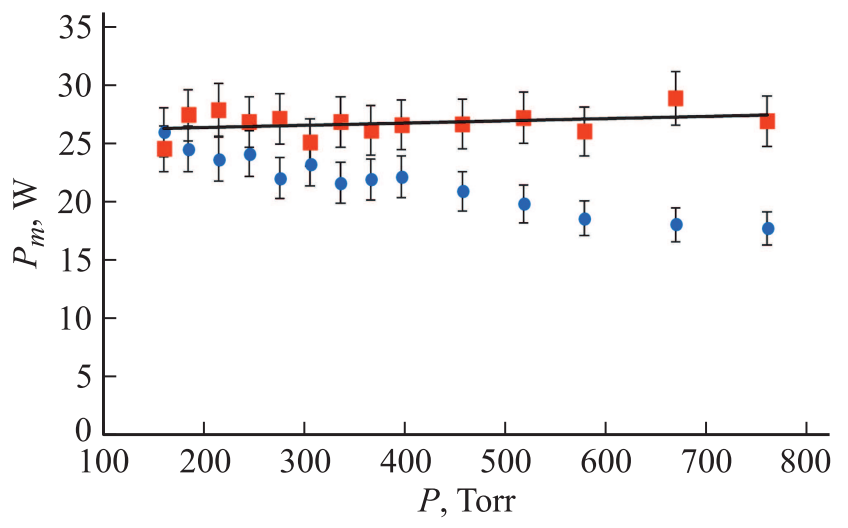

Рис. 9. Зависимости КПД (кружки) и приведенного КПД (квадратики) от давления.

свою очередь, влияет на время между микроразрядами [25], т.е. время, за которое потенциал поверхности выравнивается с потенциалом электрода. Таким образом, накопленный на поверхности заряд зависит так же от частоты, на которой происходит резонанс. Максимальный заряд, который может быть накоплен на диэлектрике, зависит от длины плазменного листа и может быть определен по формуле плоского конденсатора при заданном значении амплитуды напряжения на внешних электродах. При этом его плотность считается постоянной вне зависимости от расстояния до кромки внешнего электрода. В реальном процессе это не так. Экстремумы при давлениях 300 и 575 Torr соответствуют большему среднему по времени значению заряда, накопленного на диэлектрике, и иллюстрируют большую энергию, запасенную в конденсаторе, и соответственно большую кулоновскую силу, ускоряющую ионизированный газ синтетической струи. На рис. 9 показаны зависимости КПД $(\eta)$ и приведенного КПД ( $\left.\eta^{\prime}\right)$ от давления. Можно заметить, что приведенный КПД не зависит от давления. При этом КПД стремится к приведенному КПД при понижении давления вплоть до 150 Torr. 


\section{Заключение}

На примере простейшего линейного симметричного актуатора были определены резонансные характеристики разряда и системы в целом в зависимости от геометрии актуатора и давления воздуха. Построены резонансные кривые для симметричного актуатора с внешними электродами длиной $15 \mathrm{~cm}$ и расстоянием между ними $6.5 \mathrm{~cm}$ при различных давлениях в барокамере. Измерен КПД разряда при различных значениях давления, длины внешних электродов и фиксированной выходной мощности источника, равной $30 \mathrm{~W}$. Определены емкость и добротность системы „источник питания-актуатор-ДБР“. Проведен анализ соотношения мощности, расходуемой на создание синтетической струи, к мощности, потребляемой на вспомогательные процессы. Выполнена оценка механической мощности и построена ее зависимость от давления.

КПД разряда повышается при снижении давления в барокамере и при увеличении длины внешних электродов. При понижении давления уменьшается резонансная амплитуда напряжения на внешних электродах, таким образом, происходит перестройка системы под изменяющуюся емкость, которая существенно возрастает. При снижении давления в барокамере от $1 \mathrm{~atm}$ до 150 Torr механическая мощность увеличивается в два раза, что согласуется с двукратным уменьшением добротности и находится в согласии с проведенным анализом. Введен параметр „приведенный КПД“. Экспериментально показано, что приведенный КПД не зависит от давления. КПД сходится к приведенному КПД при снижении давления вплоть до 150 Torr.

Показано, что при уменьшении давления расход энергии на создание синтетической струи растет. Таким образом, снижение скоростного напора за счет уменьшения плотности воздуха с высотой компенсируется дополнительной энергией, которую получает синтетическая струя. Данный результат показывает возможность использования ДБР актуаторов на больших высотах, как элементов активного управления обтеканием крыла летательного аппарата.

\section{Благодарности}

Работа выполнена при поддержке программы РАН „Управление высотным летательным аппаратом при помощи диэлектрического барьерного разряда“.

\section{Конфликт интересов}

Авторы заявляют, что у них нет конфликта интересов.

\section{Список литературы}

[1] S. Baleriola, A. Leroy, S. Loyer, P. Devinant, S. Aubrun. J. Physics: Conf. Series. IOP Publishing., 753 (5), 052001 (2016).

[2] S.L. Chernyshev, M.D. Gamirullin, V.Y. Khomich, A.P. Kuryachii, V.M. Litvinov, S.V. Manuilovich, V.A. Yamshchikov. Aerospace Sci. Technol., 59, 155 (2016).

[3] A. Santhanakrishnan, J. Jacob, Y. Suzen. 3rd AIAA Flow Control Conference., 3033 (2006).

[4] C. Pinzon, R. Agarwal. 46th AIAA Aerospace Sci. Meeting and Exhibit., 559 (2008).

[5] N. Qin, H. Xia. Proceedings of the Institution of Mechanical Engineers, Part I: J. Systems Control Eng., 222 (5), 373 (2008).

[6] H. Jiang, T. Shao, C. Zhang, P. Yan, H. Liu. IEEE Transactions on Plasma Science, 46 (10), 3524 (2018).

[7] D.M. Orlov, G.I. Font, D. Edelstein. AIAA Journal, 46 (12), 3142 (2008).

[8] M. Kuhnhenn, B. Simon, I. Maden, J. Kriegseis. J. Fluid Mechanics, 809, (2016).

[9] E.A. Shershunova, M. Malashin, S. Moshkunov, V. Khomich. Acta Polytechnica, 55 (1), 59 (2015).

[10] В.М. Бочарников, И.В. Трифанов, В.В. Голуб. Письма в ЖТФ, 45 (2), 33 (2019)

[11] F.N. Glazyrin, I.A. Znamenskaya, I.V. Mursenkova, D.S. Naumov, N.N. Sysoev. Tech. Phys. Lett., 42 (1), 63 (2016).

[12] I. Biganzoli, R. Barni, A. Gurioli, R. Pertile, C. Riccardi. J. Physics: Conf. Series. IOP Publishing, 550 (1), 012039 (2014).

[13] V.R. Soloviev, V.M. Krivtsov. Plasma Sources Sci. Technol., 27 (11), 114001 (2018).

[14] I. Moralev, V. Sherbakova, I. Selivonin, V. Bityurin. Intern. J. Heat and Mass Transfer., 116, 1326 (2018).

[15] A. Starikovskiy, M. Post, N. Tkach, R. Miles 52nd Aerospace Sciences Meeting., 129, 0144 (2014).

[16] S. Dalvand, M. Ebrahimi, S.G. Pouryoussefi. Appl. Thermal Eng., 129, 50 (2018).

[17] J. Kriegseis, B. Simon, S. Grundmann. Appl. Mechanics Rev., 68 (2), (2016).

[18] В.М. Бочарников, В.В. Володин, В.В. Голуб. Письма в ЖТФ, 42 (7), 31 (2016).

[19] C. Liu, A. Fridman, D. Dobrynin. J. Physics D: Appl. Physics., 52 (10), 105205 (2019).

[20] S.B. Leonov, I.V. Adamovich, V.R. Soloviev. Plasma Sources Sci. Technol., 25 (6), 063001 (2016).

[21] A. Starikovskiy. 47th AIAA Plasmadynamics Lasers Conf., 4017 (2016).

[22] H. Yan, L. Yang, X. Qi, C. Ren. J. Physics D: Appl. Phys. 49 (29), 295203 (2016).

[23] В.М. Бочарников, В.В. Володин, В.В. Голуб. Успехи прикладной физики. 5 (6), 559 (2017).

[24] N. Benard, E. Moreau E. 6th AIAA Flow Control Conf., 3136 (2012).

[25] V.R. Soloviev J. Physics D: Appl. Phys., 45 (2), 025205 (2011). 